\title{
A categorização das funcionalidades do ambiente virtual ROODA *
}

\section{The categorization of the ROODA virtual environment funtionalities}

\author{
Caroline Bohrer do Amaral ** \\ Lúcia Barros de Souza*** \\ Maria Carolina Colombo**** \\ Patricia Alejandra Behar ${ }^{* * * *}$
}

\begin{abstract}
Resumo. Este artigo versa sobre a classificação das 19 funcionalidades do ambiente ROODA (Rede cOOperativa De Aprendizagem), um Ambiente Virtual de Aprendizagem (AVA) que integra o projeto de Educação à Distância da UFRGS. Para tanto, busca adaptar-se às necessidades e práticas pedagógicas dos docentes da universidade, e favorecer a construção do conhecimento em um espaço via web. A metodologia utilizada neste estudo foi a observação participante e a coleta de dados em versões anteriores do ROODA. As funcionalidades foram organizadas nas perspectivas de vínculo com o sistema e de relação topológica, a fim de facilitar a localização e a compreensão das mesmas.
\end{abstract}

Palavras-chaves: ambientes virtuais de aprendizagem - educação à distância funcionalidades

\begin{abstract}
This study is about the classification of 19 functionalities of ROODA (COOperative Learning Net), a Virtual Learning Environment that integrates the Project for Distance Learning from UFRGS. Our purpose is to adapt to the needs and pedagociacl practices of teachers from this university and to foster knowledge construction from the web. The methodological assumptions were gathered through participants' observations and data collection in previous ROODA versions. Functionalities are organized on the kind of link the functionality has with the environment and related to the topological disposition, in order to make their localizations and comprehension easier.
\end{abstract}

Keywords: virtual learning environment - distance learning - functionalities

\footnotetext{
* Este trabalho conta com o apoio financeiro CNPq, projeto "Desenvolvimento de Ambientes Virtuais de Aprendizagem e metodologia didático-pedagógica para Educação à Distância da UFRGS".

** Graduanda do Curso de Pedagogia da Faculdade de Educação - Universidade Federal do Rio Grande do Sul (FACED/UFRGS) - Bolsista PIBIC-CNPq - carolinebohrer82@yahoo.com.br

*** Graduanda do Curso de Pedagogia da Faculdade de Educação - Universidade Federal do Rio Grande do Sul (FACED/UFRGS) - Bolsista ITI-CNPq - daurealu@yahoo.com.br

***** Pedagoga - Licenciada pela Faculdade de Educação da Universidade Federal do Rio Grande do Sul - Professora da Rede Estadual de Ensino (RS) - mcarolcolombo@yahoo.com.br

****** Professora Adjunta do programa de Pós-Graduação em Educação e Informática na Educação - Universidade Federal do Rio Grande do Sul (PPGEdu/PGIE/UFRGS) - Coordenadora do Núcleo de Tecnologia Digital aplicada à Educação (NUTED) - pbehar@terra.com.br

V. $3 \mathrm{~N}^{\circ}$ 1, Maio, 2005
} 


\section{Introdução}

O presente artigo apresenta um estudo ${ }^{1}$ sobre a classificação das 19 funcionalidades do ambiente virtual de aprendizagem ROODA $^{2}$, a partir das perspectivas de vínculo com o sistema e de relação topológica no ambiente.

A pesquisa surgiu devido às diferentes interpretações dos membros da equipe que projetou e desenvolveu o ambiente, sobre a forma como deviam estar organizadas as funcionalidades. A equipe caracteriza-se por ser interdisciplinar, composta por sujeitos que atuam nas áreas de educação, designer e programação.

O ambiente ROODA tem uma lógica de localização das funcionalidades que diz respeito às características das mesmas. Portanto, classificar as funcionalidades do ROODA pode auxiliar os usuários na compreensão e organização do mesmo, possibilitando uma maior autonomia dentro do ambiente, explorando efetivamente seus recursos, durante o desenvolvimento das tarefas propostas à distância.

Neste estudo, define-se por funcionalidades, as ferramentas que possibilitam o desenvolvimento das disciplinas, o encontro entre os usuários e a valorização do processo de cooperação.

\section{2. $O$ ambiente virtual de aprendizagem ROODA}

Para entender a classificação das funcionalidades, é necessário descrever o ambiente ROODA, a fim de ilustrar a caminhada percorrida e as características pedagógicas do mesmo.

O ROODA - Rede cOOperativa De Aprendizagem, disponível em http://homer.nuted.edu.ufrgs.br, foi criado em $2000^{3}$ pelo Núcleo de Tecnologia Digital aplicada à Educação da Faculdade de Educação da Universidade Federal do Rio Grande do Sul. Desde então, o ambiente vem sofrendo modificações, visando a melhoria e a ampliação de suas possibilidades de uso. É um software livre que integra a tecnologia digital com a educação. Caracteriza-se como um ambiente de aprendizagem interativo que disponibiliza funcionalidades síncronas e assíncronas via Web para oportunizar a interação/comunicação entre os sujeitos.

Este ambiente virtual de aprendizagem (AVA), desde o início de seu desenvolvimento, tem como particularidade ser centrado no usuário ${ }^{4}$. Esta característica propicia uma maior interação entre os sujeitos e as diferentes disciplinas, pois não é necessário que o usuário tenha de efetuar o logout (saída) cada vez que deseje visualizar ou simplesmente, consultar outra disciplina.

O ambiente foi desenvolvido de forma a viabilizar a lógica de navegação pelo mesmo. Além de distribuir os ícones de acesso às funcionalidades em locais distintos (menu superior, abas laterais e área de trabalho), devido aos dois diferentes vínculos das ferramentas com o sistema (geral e específico), preocupou-se também com os nomes das funcionalidades. Estes, por sua vez, objetivam explicitar a função das funcionalidades.

O ROODA foi reconhecido institucionalmente a partir de 2003 e passou a fazer parte do projeto de Educação à Distância da UFRGS, como uma das plataformas oficiais de aprendizagem. Conseqüentemente, foi preciso (re)construir todas as suas funcionalidades e sua interface gráfica, com o objetivo de adaptar-se às necessidades e práticas pedagógicas dos docentes da universidade, favorecendo a construção do conhecimento em um espaço via Web. Durante o ano de 2005, ele está em período de testes e avaliação pela instituição. 
O ambiente foi modelado em UML, construído com GNU/LINUX e programado em linguagem PHP. A equipe desenvolvedora buscou tornar o ROODA o mais aberto possível para que o próprio professor possa ativar/desativar as funcionalidades desejadas de acordo com a sua prática. Desta forma, valoriza-se o "modelo educacional" de cada docente, isto é, sua metodologia e paradigma. Também, se oferece a possibilidade ao usuário de personalizar seu ambiente. Para tanto, foram planejados três diferentes temas de interface gráfica, selecionados de acordo com as preferências/necessidades dos usuários.

\subsection{Os diferentes temas da interface gráfica}

A interface do ROODA deseja tanto oferecer uma agradável experiência estética, quanto permitir uma navegação intuitiva e rápida. Considerando-se questões de adaptabilidade e usabilidade, o ambiente pode ser montado e customizado pelo professor segundo seus objetivos educacionais. Os usuários encontram três temas de interface disponíveis para uso: Fotográfica (Figura 1), Aqua (Figura 2) e Grafite (Figura 3). Os temas apresentam imagens, textos e botões exatamente nas mesmas posições e seguem os critérios da classificação propostos nesta pesquisa para o ambiente.

As imagens foram tratadas a fim de facilitar seus carregamentos. Este tipo de design tem como objetivo facilitar a navegação pelo ambiente, oferecendo formas diversas de acesso às funcionalidades, além de facilitar a integração das mesmas. Preocupou-se, também, com a maximização da usabilidade, a fim de diminuir o número de "cliques" necessários para acessar as diferentes ferramentas e informações. O ROODA traz ainda, ajuda ${ }^{5}$ para cada uma das telas.

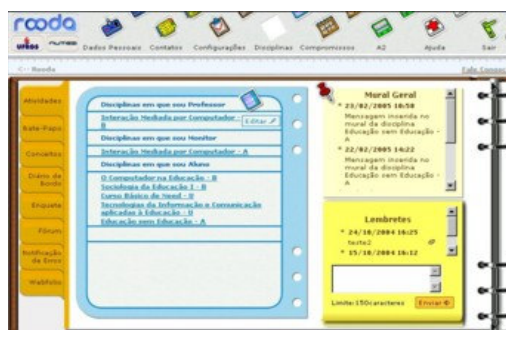

Figura 1 - Interface com tema "Fotográfica"

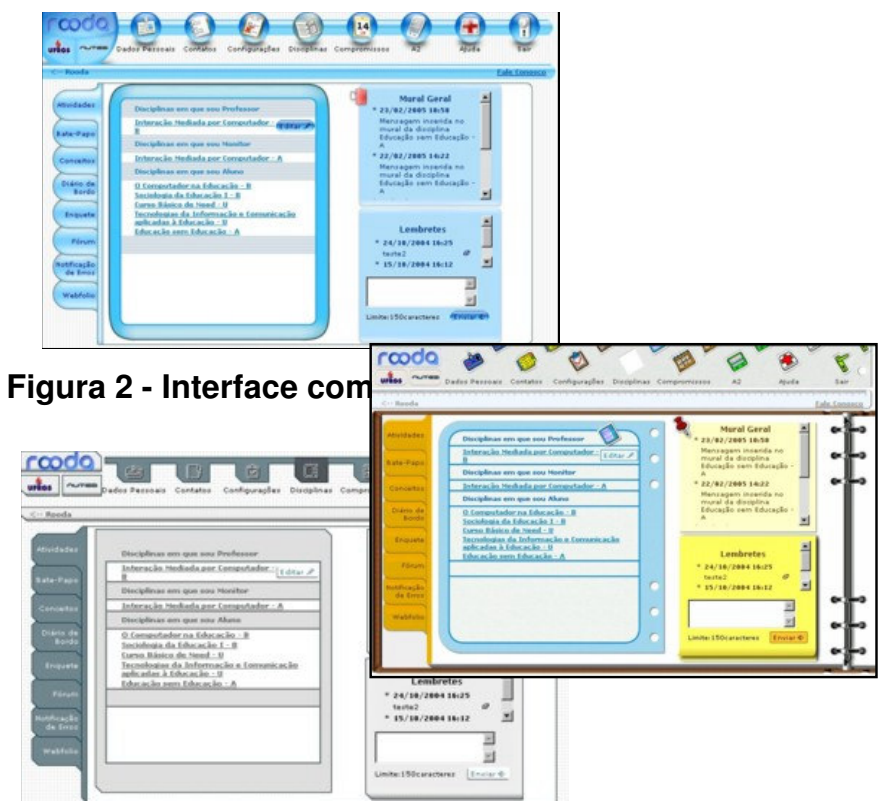

Figura 3 - Interface com tema "Grafite"

\subsection{As funcionalidades do ambiente ROODA}

A partir das pesquisas realizadas com as versões anteriores do ROODA ${ }^{6}$ e com softwares livres disponíveis na Internet, algumas funcionalidades presentes nas versões anteriores foram reformuladas e outras novas foram desenvolvidas. Na nova versão do ROODA, tem-se um total de 19 funcionalidades, conforme descritas a seguir:

1. A2: possibilita a comunicação síncrona entre os sujeitos conectados no ROODA;

2. Atividades: oportuniza a montagem/organização de atividades/aulas; 
3. Bate-papo: permite a comunicação síncrona entre os usuários, consultas em salas salvas no ROODA e salvá-las no computador do usuário;

4. Biblioteca: disponibiliza artigos, trabalhos e apresentações que serão utilizados como material de apoio das disciplinas e do próprio ambiente;

5. Compromissos: oportuniza a organização cronológica de compromissos;

6. Conceitos: possibilita ao o professor registrar os conceitos/notas dos alunos, ao longo de todo o semestre, e postar comentários;

7. Configurações: possibilita ao usuário configurar as demais funcionalidades do ROODA de acordo com as preferências do usuário, alterando o padrão;

8. Contatos: lista nome e e-mail de outros usuários do ROODA, possibilitando o envio de mensagens para estes;

9. Dados Pessoais: disponibiliza informações pessoais sobre o usuário;

10. Diário de Bordo: possibilita aos usuários o registro das construções, inquietações, observações, etc. Tem-se a possibilidade de postar mensagens e inserir comentários;

11. Disciplinas: apresenta as informações sobre determinada disciplina;

12. Enquete: possibilita a realização de pesquisas de opinião com pergunta e respostas;

13. Fórum: permite o debate de temas através de comunicação assíncrona. As trocas são organizadas em forma linear, no padrão PHPBB;

14. Gerência da Disciplina: possibilita a ativação, o registro de dados e a habilitação de funcionalidades pelo professor;

15. Lembretes: possibilita ao usuário fazer anotações para uso pessoal;

16. Lista de Discussão: organiza uma lista de endereços eletrônicos de alunos/professores de uma mesma disciplina, sendo que as mensagens são encaminhadas aos endereços de e-mail cadastrados no ROODA;

17. Mural: apresenta recados enviados pelo sistema, no Mural Geral e possibilita a visualização de recados relativos à disciplina, enviados pelo professor, no Mural Disciplina;

18. Produções: oferece recursos disponibilizados pelo ambiente para a construção coletiva de trabalhos. Promove um espaço para a construção de um projeto e/ou trabalho individual ou coletivo; Diário da Produção: registro do processo do grupo;

19. Webfólio: propicia a publicação e a organização de arquivos a serem acessados no formato de páginas para Web, a fim de socializar as mesmas.

Uma particularidade deste ambiente é a integração das funcionalidades. Esta possibilidade objetiva trabalhar os objetos de estudo de forma contextualizada através de recursos oferecidos pelo sistema. Logo, busca-se favorecer uma hipertextualidade ao que é produzido/debatido dentro do ROODA, com links apontando para diferentes espaços em que se está abordando o objeto de estudo em questão. A integração poderá ser feita pelo professor (ao habilitar as funcionalidades) entre as seguintes funcionalidades:

- Enquete -> Fórum e Atividade;

- Biblioteca -> Fórum e Atividade; 
- Atividade -> Bate-Papo, Conceito, Fórum, Lista de Discussão, Produção, Biblioteca e Enquete;

- Produção -> Bate-Papo, Diário da Produção, Fórum, Lista de Discussão, Biblioteca, Enquete e Atividade.

\section{A pesquisa}

\subsection{Metodologia}

A metodologia utilizada neste estudo foi a observação participante e a coleta de dados na versão 0.7 do ROODA (anterior à atual).

A observação participante, segundo Gil (1985), caracteriza-se pelo fato de que o pesquisador pode pertencer à comunidade que investiga, facilitando o acesso aos dados. Sendo assim, a equipe do NUTED, mais especificamente, o grupo responsável pelo design pedagógico do ambiente ROODA, observou-se de maneira participativa a construção do ambiente fazendo relações com suas versões anteriores.

Logo, a partir desta coleta de dados são apresentadas duas perspectivas iniciais para a classificação das funcionalidades no ambiente ROODA: de vínculo com o sistema e de relação topológica.

\subsection{Resultados e Discussões}

A perspectiva de vínculo com o sistema é categorizada em geral e específica. A categoria geral possui 11 funcionalidades disponíveis a todos os usuários. Destas, quatro podem ou não ser habilitadas à disciplina. Sendo elas: Fórum, Mural, Produções e Webfólio. A categoria específica é constituída por 8 funcionalidades, acessadas quando vinculadas a uma disciplina. Sendo elas: Atividades, Bate-papo, Biblioteca, Conceitos, Enquete, Gerência da Disciplina, Diário de Bordo e Lista de Discussão.

Esta categorização parte da concepção de usabilidade das funcionalidades, relacionando-se ao caminho que o usuário pode estabelecer durante a navegação pelo ambiente. Para que seja possível este tipo de relação, o sujeito precisa conhecer as características de cada funcionalidade, ou seja, se ela está disponível para qualquer usuário (geral) ou se ela pode ser visualizada quando selecionada pelo professor na Gerência da Disciplina (específica).

A outra perspectiva, a de relação topológica, é baseada na disposição dos links para acesso às funcionalidades no ambiente, a partir da concepção de espaço. Independente do link em que clicar, a visualização das informações irá se dar na área de trabalho do ambiente (mesma tela) ou em uma nova janela (popup). As funcionalidades são organizadas em menu superior, abas laterais e área de trabalho (Figura 4). 


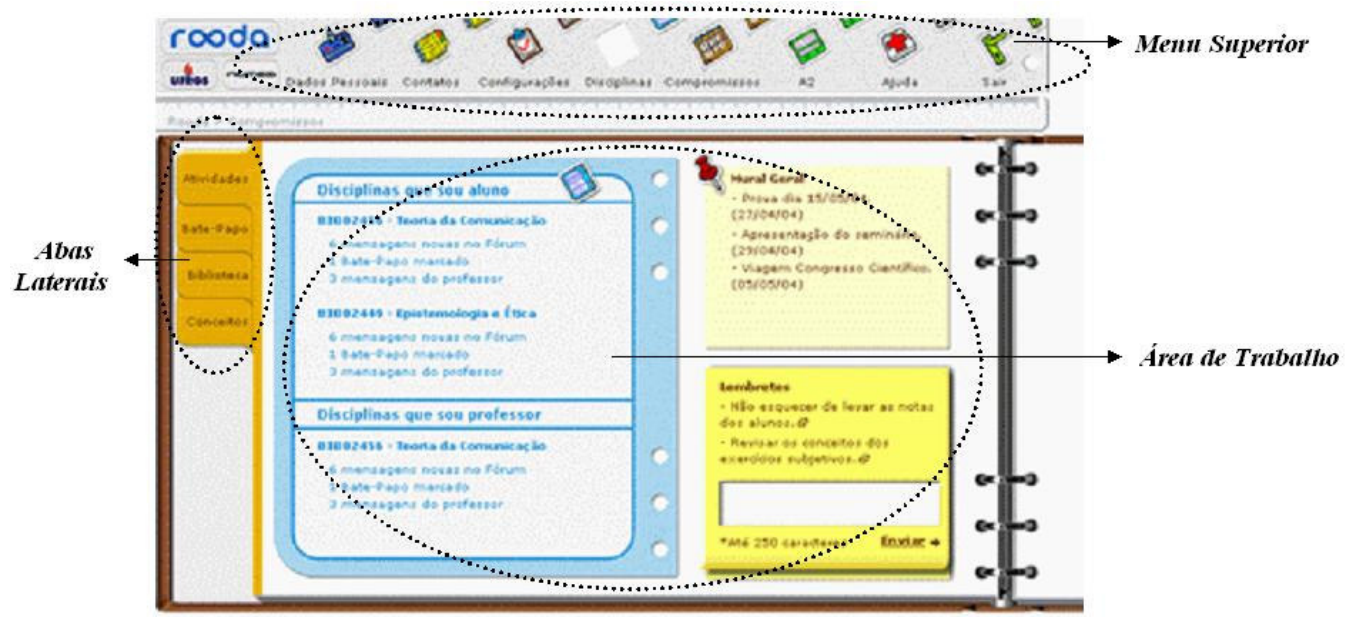

Figura 4 - Relação Topológica

No menu superior encontram-se 6 funcionalidades e o recurso Ajuda. Sendo elas: Dados Pessoais, Contatos, Configurações, Disciplinas, Compromissos e A2. Nas abas laterais visualizam-se até 10 funcionalidades, dependendo da seleção de funcionalidades realizada pelo professor. $\mathrm{Na}$ área de trabalho pode-se observar até três funcionalidades. Sendo elas: Mural, Lembretes, Disciplinas.

Essa perspectiva é relacionada à disposição das funcionalidades no ambiente, independente do tema de interface. Assim, o sujeito navega pelo ROODA, não precisando ter a noção de funcionalidades gerais e específicas (vínculo com o sistema) para encontrá-las. Tal classificação baseia-se em princípios topológicos (relações espaciais), o que pode incluir as correspondências entre a parte e o todo (Lewin, 1973) e nos estudos sobre noções topológicas realizados por Piaget (1993).

Este AVA pode ser considerado um espaço aberto que necessita de senha e login para o acesso por parte do usuário. É conexo, já que o sujeito visualiza as funcionalidades nas três disposições topológicas; estas possuem relação umas com as outras para propiciar espaços para a argumentação de suas opiniões, sugestões e idéias. Como exemplo, a funcionalidade Enquete que pode ser vinculada a um tópico do Fórum para que os sujeitos debatam sobre o assunto em pauta.

Lewin (1973, p. 125) argumenta que há "a possibilidade de locomoções de cada ponto para outro ponto, por caminhos que se situam totalmente dentro da região". Portanto, o sujeito navega de uma funcionalidade para outra, mantendo-se dentro do ROODA. Para isso, é necessário somente um login, independente de quantas disciplinas esteja cursando através do ambiente. A perspectiva de relação topológica auxiliará o sujeito a estabelecer uma conexão entre um determinado ponto do ROODA e sua respectiva funcionalidade.

A categorização das funcionalidades encontra-se ilustrada na tabela abaixo (Tabela 1). Esta apresenta as funcionalidades classificadas nas perspectivas de vínculo com o sistema e de relação topológica, bem como suas respectivas categorias.

Tabela 1 - Tabela organizacional da categorização

\begin{tabular}{|l|l|c|c|c|}
\hline \multirow{2}{*}{ FUNCIONALIDADES } & \multicolumn{3}{|c|}{ PERSPECTIVAS } \\
\cline { 2 - 4 } & \multicolumn{2}{|c|}{ Vínculo com o Sistema } & \multicolumn{2}{|c|}{ Relação Topológica } \\
\cline { 2 - 5 } & Geral & Específica & Menu superior & Abas Laterais Área de Trabalho \\
\hline
\end{tabular}




\begin{tabular}{|c|c|c|c|c|c|}
\hline CINTED-UI & & & & \multicolumn{2}{|c|}{ Novas Tecnologias na Educação } \\
\hline A2 & $x$ & & $x$ & & \\
\hline Atividades & & $\mathrm{x}$ & & $x$ & \\
\hline Bate-Papo & & $\mathrm{X}$ & & $\mathrm{X}$ & \\
\hline Biblioteca & & $\mathrm{x}$ & & $\mathrm{x}$ & \\
\hline Compromissos & $\mathrm{X}$ & & $\mathrm{x}$ & & \\
\hline Conceitos & & $\mathrm{x}$ & & $\mathrm{x}$ & \\
\hline Configurações & $\mathrm{X}$ & & $\mathrm{X}$ & & \\
\hline Contatos & $\mathrm{X}$ & & $\mathrm{x}$ & & \\
\hline Dados Pessoais & $\mathrm{X}$ & & $\mathrm{X}$ & & \\
\hline Diário de Bordo & & $\mathrm{x}$ & & $\mathrm{x}$ & \\
\hline Disciplina & $\mathrm{x}$ & & $x$ & & $x$ \\
\hline Enquête & & $\mathrm{X}$ & & $\mathrm{X}$ & \\
\hline Fórum & $x$ & $\mathrm{x}$ & & $x$ & \\
\hline Gerência da Disciplina & & $\mathrm{x}$ & & & $x$ \\
\hline Lembretes & $x$ & & & & $x$ \\
\hline Lista de Discussão & & $\mathrm{X}$ & & $\mathrm{x}$ & \\
\hline Mural & $\mathrm{x}$ & $\mathrm{x}$ & & & $x$ \\
\hline Produções & $x$ & $\mathrm{X}$ & & $\mathrm{x}$ & \\
\hline Webfólio & $\mathrm{x}$ & $\mathrm{x}$ & & $\mathrm{x}$ & \\
\hline
\end{tabular}

\section{Palavras não finais...}

A partir da análise destas duas perspectivas, foi possível perceber que ambas se entrelaçam em determinados momentos de uso do ambiente. Esta relação pode ser observada conforme a seleção das funcionalidades por parte do professor. Dois exemplos podem ser encontrados na funcionalidade Fórum: (1) ao clicar na aba Fórum, antes de acessar uma disciplina, o usuário pode ter acesso aos fóruns gerais e aos específicos na área de trabalho; (2) ao acessar uma disciplina, e após clicar na aba Fórum, o usuário tem acesso ao conteúdo do fórum específico na área de trabalho.

Outra modificação que ocorre na disposição das funcionalidades pode ser observada nas abas laterais. Conforme a escolha da disciplina por parte do usuário, a ordem das abas será modificada, já que os professores terão a opção de escolher as funcionalidades, na Gerência da disciplina, que mais se adequam à perspectiva pedagógica de cada educador. Apesar das abas serem alteradas de acordo com a disciplina, estas são mantidas em ordem alfabética para facilitar a localização.

Pretende-se que o ROODA possa ser utilizado na UFRGS, atendendo as demandas de professores/alunos, em nível de Graduação, Pós-Graduação, Especialização e Extensão, e que também transcenda "as paredes" desta Universidade, estendendo-se para outras instituições superiores e escolas de Ensino Fundamental e Médio.

A partir da utilização da plataforma durante o presente ano, a equipe do NUTED irá voltar seu olhar para a repercussão dessa categorização junto aos usuários, a nível avaliativo, assim como as diferentes interpretações que poderão ser estabelecidas por estes. A partir deste olhar, poder-se-á obter um retorno da usabilidade do sistema e possíveis mudanças/alterações a serem realizadas no que se refere ao aperfeiçoamento contínuo do ambiente. 
A primeira coleta de dados será realizada no semestre 2005/01, através de onze disciplinas que serão ministradas em cinco cursos de graduação e uma de pósgraduação, além do curso de capacitação para os professores/técnicos administrativos da UFRGS.

Como a nova versão do ROODA conta com o número de dezenove funcionalidades, as classificações abordadas neste artigo e as novas a serem estabelecidas pelos usuários, facilitarão a navegação pelo ambiente. Essas poderão ser utilizadas como referência para possíveis reorganizações da interface.

Esta pesquisa poderá auxiliar outras equipes de planejamento/desenvolvimento de ambientes virtuais, pois poderão valer-se deste princípio de categorização para proporcionar espaços na Web que sejam motivadores para a aprendizagem e projetados de modo a favorecer a navegação, seguindo uma lógica, e a interação entre os usuários.

Dando seguimento a esta pesquisa, iniciar-se-ão os estudos em relação à Ergonomia de Software. O propósito será identificar os dispositivos que possam proporcionar eficácia, conforto e segurança para a navegação e uso do ROODA, de acordo com os diferentes perfis de usuários.

\section{Notas de texto}

1. Agradecimento à Doutoranda do Programa de Pós-Graduação de Informática na Educação Silvia Meirelles Leite pelo constante apoio durante a realização deste estudo.

2. Informações disponíveis em http://www.nuted.edu.ufrgs.br

3. O Projeto ROODA ganhou o prêmio do Programa de Apoio à Pesquisa em Educação a Distância - PAPED2002 da CAPES/SEED/MEC. Foi um dos cinco trabalhos selecionados em todo o país sobre materiais didáticos multimídia para uso em cursos ou disciplinas, ministrados presencialmente ou a distância, via Internet, elaborados por professores do ensino superior de graduação e pós-graduação strictu sensu e destinados à aprendizagem de disciplinas, conjunto de disciplinas ou temas transversais dos currículos do Ensino Básico (disponível em http://www.mec.gov.br/seed/paped).

4. Também existem ambientes centrados no curso, como o Teleduc (http://teleduc.nied.unicamp.br). Neste caso, o usuário precisa deslogar-se de uma disciplina para acessar outra, podendo utilizar apenas os recursos e serviços oferecidos pela mesma.

5. Ajuda: recurso no ROODA/UFRGS que fornece instruções para auxiliar o usuário na navegação e na utilização desse ambiente.

6. Um detalhamento das versões anteriores do ROODA é encontrado em Behar, 2001a e $2001 b$.

\section{Referências Bibliográficas}

BEHAR, Patricia. ROODA - Rede cOOperativa De Aprendizagem - Uma plataforma de suporte para aprendizagem à distância. Em pauta - Revista Informática na Educação - Teoria \& Prática, Porto Alegre, v. 4, n. 2, p. 87 - 96, dez./2001. A caminho de um ambiente de aprendizagem à distância - ROODA - Rede cOOperativa De Aprendizagem, In: XII SIMPÓSIO BRASILEIRO DE INFORMÁTICA NA EDUCAÇÃO - XII SBIE, 2001, Vitória. Anais. Vitória: Sociedade Brasileira de Computação, nov./2001, p.168-175. 
- Construyendo una interface para el ambiente virtual - ROODA - Rede cOOperativa De Aprendizagem. Em pauta - Revista Informática na Educação Teoria \& Prática, Porto Alegre, v.6, n.1, p.23 - 40, 2003.

Em busca das interações interindividuais no ROODA, Em pauta - Revista de Educação da PUCRS. Porto Alegre, n. 1 (52), p. 169 - 199, jan/abril, 2004.

. Projeto ROODA: a participação das mulheres no desenvolvimento e utilização de ferramentas de software livre, In: V FÓRUM INTERNACIONAL DE SOFTWARE LIVRE, 2004, Porto Alegre. Anais. Porto Alegre. Apoio: Sociedade Brasileira de Computação, 2004, p.257.

GIL, Antônio Carlos. Métodos e técnicas da pesquisa social. São Paulo: Atlas, p. 105$112,1985$.

LEWIN, Kurt. Princípios de Psicologia Topológica. São Paulo: Cultrix, 1973.

PIAGET, Jean. Estudos Sociológicos. Rio de Janeiro: Forense, 1973.

NIELSEN, Jakob. Projetando Websites. Editora: Campus, first edition, 2000.

PIAGET, J., INHELDER, B. A representação do espaço na criança. Porto Alegre: Artes Médicas, 1993.

RUBIN, Jeffrey. Handbook of Usability Testing: How to Plan, Design, and Conduct Effective Tesbts, Wiley \& Sons, New York, first edition, 1994. 